\title{
Nano-structuring of molybdenum and tungsten surfaces by low-energy helium ions
}

\author{
G. De Temmerman, ${ }^{1, a)}$ M. Balden, ${ }^{2}$ K. Bystrov ${ }^{3}$ C. Arnas, ${ }^{4}$ L. Marot, ${ }^{5}$ G. Matern, ${ }^{2}$ and
} J.J. Zielinski ${ }^{3}$

1) FOM Institute DIFFER, Dutch Institute For Fundamental Energy Research, Association EURATOM-FOM, Trilateral Euregio Cluster, P.O. Box 120\%, 3430 BE Nieuwegein, The Netherlands

2) Max Planck Institute for Plasma Physics, EURATOM Association, 85748 Garching, Germany

3) FOM Institute DIFFER, Ducth Institute For Fundamental Energy Research, Association EURATOM-FOM, Trilateral Euregio Cluster, P.O. Box 120\%, 3430 BE Nieuwegein, The Netherlands

4) Laboratoire PIIM, CNRS/Aix-Marseille Universit, 13397 Marseille, France

5) Department of Physics, University of Basel, Basel, CH-4056, Switzerland

(Dated: 7 November 2012; Revised 7 November 2012)

The formation of metallic nanostructures by exposure of molybdenum and tungsten surfaces to high fluxes of low energy helium ions is studied as a function of the ion energy, plasma exposure time, and surface temperature. Helium plasma exposure leads to the formation of nanoscopic filaments on the surface of both metals. The size of the helium-induced nanostructure increases with increasing surface temperature while the thickness of the modified layer increases with time. In addition, the growth rate of the nano-structured layer depends on the surface temperature, too. The size of the nanostructure appears linked with the size of the near-surface voids induced by the low energy ions. The results presented here thus demonstrate that surface processing by low-energy helium ions provides an efficient route for the formation of porous metallic nanostructures.

a) Electronic mail: g.c.detemmerman@differ.nl 


\section{INTRODUCTION}

The paramount importance of nanoparticles and nanostructured surfaces in various fields of material-related research stems from the unique properties of these surfaces. In particular, nano-structured surfaces with large porosities are very benefitial for photo-catalytic processes such as the photo-electrochemical splitting of water for example ${ }^{1,2}$. In addition, there is a growing interest for the development of black metals i.e. nanostructured metals allowing maximum light absorption for solar power concentration where refractory metals are of interest because of their high melting points ${ }^{3}$.

Bombardment of metallic surfaces by energetic ions (keV range) is known to lead to strong microstructural changes such as dislocation loops, voids, holes, etc. It has been observed recently that strong morphology changes, such as blister formation, can also occur on metal surfaces when the ion energy is below the threshold for atom displacement ${ }^{4}$. This effect is especially pronounced in the case of low-energy helium ions. Tungsten surfaces exposed to large fluences of low energy $(<60 \mathrm{eV})$ helium ions are found to exhibit a nanostructured surface morphology consisting of nanometric filaments ${ }^{5,6}$. Detailed studies have been conducted to elucidate the formation mechanism of such structures ${ }^{7,8}$ which appears linked to the formation and coalescence of helium bubbles in the near-surface region inducing swelling of the surface. The helium-induced nanostructure in these studies is characterized by a very low density of less than $10 \%$ of that of the bulk material ${ }^{9}$ and hence a high level of porosity causing a total blackening the surface completely. In turn, the surface reflectivity has been measured ${ }^{11}$ to be as low as $1 \%$ across the whole visible spectrum making such surfaces ideal for optimum light absorption. There are some indications that this process is not exclusive to tungsten but that other metals such as molybdenum could be used to produce similar structures ${ }^{11}$.

The present article compares the development of helium-induced nanostructures on molybdenum and tungsten under similar conditions. The evolution of the surface morphology as a function of plasma exposure time, surface temperature and ion energy will be discussed. 


\section{EXPERIMENTAL}

Polycrystalline tungsten and molybdenum samples were exposed to pure helium plasmas in the Pilot-PSI linear plasma generator. The plasma is generated by a so-called cascaded arc source and exhausts into the vacuum vessel. An axial magnetic field is used to confine the plasma and generate an intense magnetized cylindrical plasma beam. The experimental setup is described in more details elsewhere ${ }^{12}$. The magnetic field was fixed at $0.4 \mathrm{~T}$ and can be maintained for a duration of $150 \mathrm{~s}$. Discharge currents in the range 150-250 A and gas flows in the range $1.4-2 \mathrm{slm}\left(1\right.$ standard litre per minute $\left.=4.48 \times 10^{20} \mathrm{part} \cdot \mathrm{s}^{-1}\right)$ were used in this study. The plasma parameters are measured by means of a Thomson scattering (TS) system $^{13}$ located at a distance of $17 \mathrm{~mm}$ from the plasma-exposed surface. The plasma density and temperature exhibit Gaussian profiles with a Full-Width Half Maximum (FWHM) of about $10 \mathrm{~mm}$. Plasma densities and temperature were in the range $0.4-3 \times 10^{20} \mathrm{~m}^{-3}$ and 1.3-3 eV respectively. Figure 1 shows typical profiles of electron densities and temperature during a helium discharge used in the experiments presented here. The ion flux to the surface is determined from the Thomson scattering measurements by assuming that ions are accelerated over the pre-sheath up to the sound speed ${ }^{14}$. In addition, ion fluxes determined from ion saturation current measurements are found to be in good agreement with the TS measurements. The ion fluxes used in this study were in the range $0.7-3 \times 10^{24} \mathrm{~m}^{-2} \mathrm{~s}^{-1}$ or $11-48 \times 10^{4} \mathrm{~A} \cdot \mathrm{m}^{-2}$.

Plasma-exposed samples were made of polycrystalline tungsten and molybdenum cut from a rod. After polishing to a mirror finish, the samples were ultrasonically cleaned in aceton and alcohol, and outgassed at $1000^{\circ} \mathrm{C}$ for $15 \mathrm{~min}$. The samples are clamped on a water-cooled copper holder, an intermediate layer of Grafoil is used to improve the thermal contact. Note that the plasma-exposed surface has a diameter of $22 \mathrm{~mm}$ which is larger than the FWHM of the plasma beam hence the surface temperature has a Gaussian profile. The peak surface temperature is measured by a multi-wavelength pyrometer (FMPI SpectroPyrometer, FAR associates) which measures in the wavelength range 900-1600 nm f, while the 2D surface temperature profile is measured by a fast infrared camera (FLIR SC7500MB) with a typical frame rate of $100 \mathrm{~Hz}$. The surface emissivity is determined by cross-calibration with the pyrometer measurements. The pyrometer measures the light emitted from the sample in the range 900-1600 $\mathrm{nm}$. The surface temperature is then determined 
by a fit of the measured spectrum and comparison with the Planck's curve. The surface emissivity is then determined by comparing the measured photon fluxes to what would be expected from a black-body. The emissivity value for the infrared camera is then adjusted so as the temperature determined from the IR camera matches that from the pyrometer. In general, the resulting emissivity value is observed to be very close to that determined by the pyrometer. Detailed heat load calculations have shown that this technique yields a good agreement with calorimetric measurements of the plasma-deposited power ${ }^{15}$. The surface temperature is coupled with the plasma-deposited power and is controlled by varying the plasma conditions. Surface temperatures in the range $600-2000^{\circ} \mathrm{C}$ were used. After plasma exposure, the samples are analysed by Scanning Electron Microscopy (SEM), top view and cross-section, and Energy Dispersive X-Ray spectroscopy (EDX). Tungsten samples were analysed with a Hitachi S-4800 field emission microscope with $5 \mathrm{kV}$ acceleration voltage. Cross-sectioning of the samples was carried out by mechanical cleavage, the sectioned surface was then observed without further preparation. Molybdenum surfaces were observed using a Helios NanoLab 600 microscope. Cross-sectioning of the samples was done in-situ using a focused $30 \mathrm{keV}$ gallium ion beam.

\section{RESULTS AND DISCUSSION}

During plasma exposure the surface temperature is monitored by a spectropyrometer, which allows an in-situ study of the evolution of the surface emissivity. Figure 2 shows the temporal evolution of the peak surface temperature and emissivity (at the location of the maximum temperature) of a molybdenum and tungsten surface during successive plasma exposures (delimited by dashed lines). In both cases, the initial emissivity is relatively low because of the mirror-finish polishing but increases very fast during the first 50-100s of plasma exposure. In the case of tungsten (fig. 2a), the emissivity increases from 0.07 to 0.29 within the first plasma exposure and remains almost constant during the following exposure. Note that the value of the emissivity is not absolute as it accounts for the optical system transmission and instrument factors. For molybdenum (fig. 2b), only a slight increase of the emissivity is measured during the first plasma pulse but the temperature varies strongly during the pulse; note that the signal was too low during the first 50 seconds of plasma exposure to get a reliable signal. In the following pulse, the emissivity rises very fast (factor 
2) within about 20 seconds and then remains constant during the remainder of the exposure. It is interesting to note that the initial emissivity values during the second exposure correspond to the final emissivity value obtained during the first exposure, indicating an incremental surface modification. The emissivity remains almost constant during the 3rd exposure- the discontinuity in the data between 150 and $180 \mathrm{~s}$, is caused by an automatic setting change after saturation of the pyrometer. After exposure, both samples showed a total blackening of the surface. Those observations are consistent with the observed darkening and decreased reflectivity of tungsten surfaces after exposure to pure helium plasmas, which has been reported previously ${ }^{6,10,16}$. The current results demonstrate that the surface blackening occurs on a very short timescale, for fluences higher than $10^{25} \mathrm{~m}^{-2} \mathrm{~s}^{-1}$.

Figure 3 shows SEM pictures of molybdenum surfaces after exposure to a helium plasma for $500 \mathrm{~s}$, corresponding to a fluence of $3 \times 10^{26} \mathrm{~m}^{-2}$, and with a surface temperature of $1000^{\circ} \mathrm{C}$ and two different ion energies- 25 and $45 \mathrm{eV}$. The observed surface modifications strongly depend on the impinging ion energy. For $\mathrm{E}_{i o n}=25 \mathrm{eV}$, the morphology changes appear to be dependent on the grain orientation as illustrated in fig. 3a where sharp boundaries between different surface structures are clearly observed. Fig. 3b and c show high magnification pictures of those different structures. While large pore formation is common to these two areas, the pore shape is very different. Both circular (fig. 3b) and elongated pores (fig. 3c) are visible. In some cases, pinholes can be found at the bottom of the elongated voids. When the ion energy is increased to $45 \mathrm{eV}$, the surface is covered with nanoscopic filaments whose diameter is in the range 20-50 nm (fig. 3d). The plasma-exposed surface then appears homogeneous with no signs of the grain-dependent behaviour observed at lower ion energy. Figure 4 illustrates the surface modifications induced by high fluxes of low energy helium ions on tungsten substrates with a temperature of $1000^{\circ} \mathrm{C}$. The experimental conditions were similar to those used for the molybdenum samples. Here also, for the lowest ion energy, the appearance of the modified surface is dependent on the tungsten grain orientation (fig. 4a). In the two cases, many pinholes are observed on the surface with sizes below $50 \mathrm{~nm}$. In fig. $4 \mathrm{~b}$, irregularly-shaped structures are seen protruding from the surface. In other locations (fig. 4c), a wavy structure is observed with also protruding filaments. It is interesting to note that although the surface modifications in both cases are rather different, the shape of the protusions in both cases is similar. At higher ion energies, a fully-developed nano-structure is formed (fig. 4d). 
The effect of the helium ion energy on the surface modifications of tungsten was investigated in details $\mathrm{in}^{17}$. It was found that below $27 \mathrm{eV}$ surface modifications could hardly be detected (by SEM) while an ion energy of 32-37 eV was necessary to observe protrusion of nanostructure from the surface. Similarly, it is reported in ${ }^{7}$ that below $20 \mathrm{eV}$, only voids formation on the surface can observed but that protruding filaments were absent. Our data confirm the strong influence played by the ion energy on the helium-induced surface modification process. It appears that increasing the ion energy from 25 to $45 \mathrm{eV}$ accelerates strongly the formation rate of the nano-filaments although nano-filament formation is already observed at $25 \mathrm{eV}$. Ion-produced damages can be excluded since the ion energy in this study is well below the expected threshold $(\approx 0.5 \mathrm{keV})$ for helium on tungsten. An increase of the ion energy from $25 \mathrm{eV}$ to $45 \mathrm{eV}$ will modify the ion penetration depth and the particle reflection coefficient i.e. the helium trapping in the surface- which has been experimentally measured in ${ }^{17,19}$. In addition, it has been previously shown ${ }^{20}$ that the development rate of helium-induced nanostructures on tungsten increases with the incoming ion flux and saturates for a helium flux of $10^{22} \mathrm{~m}^{-2} \mathrm{~s}^{-1}$, implying that a given helium concentration in the near-surface is needed for the morphology changes to occur.

Figure 5 shows the morphology of tungsten surfaces exposed to similar plasma conditions to those in fig. 4 but with a surface temperature of $1400^{\circ} \mathrm{C}$. Filament protrusion is clearly observed even for the lowest ion energy. A strong surface roughening together with the presence of numerous pinholes can be seen in fig. $5 \mathrm{a}$ and b. It is rather remarkable that some of those protrusions appear to be barely attached to the surface. At the same time, some protrusions are forming bridges and/or have bended shapes. In general the protrusions do not exhibit particular alignment with respect to the surface, which would indicate that the formation process is independent on the electric field present in the electrostatic sheath. The height distribution of the filaments is relatively wide as can be observed from fig. $5 \mathrm{~b}$. Comparison of fig. 5b and d points out once again to the strong influence of the ion energy on the surface modification kinetics. While at $25 \mathrm{eV}$ the filament height is around $100 \mathrm{~nm}$ and the surface coverage relatively low, at $45 \mathrm{eV}$ a full coverage of the surface is obtained and the thickness of the plasma-modified surface is in the range 500-800 $\mathrm{nm}$. The crosssection images (fig $5 \mathrm{c}$ and d) clearly evidence the presence of large voids in the near-surface region. In addition, voids extending in different directions and with inner diameter of up to $100 \mathrm{~nm}$ can be observed. It therefore appears as if the surface protrusions are related 
to the surface swelling induced by the growth of the near surface bubbles ${ }^{7}$. The protruding structures appear to present facetted surfaces on their top part (fig. 5d).

The effect of the surface temperature on the helium-induced surface modification is further investigated in fig. 6 for molybdenum surfaces. In this case, the ion energy was $45 \mathrm{eV}$ and the exposure time was $1000 \mathrm{~s}$. Both top-view and cross section images are shown. Evidently, the surface temperature has a strong effect on the resulting surface morphology. At $620^{\circ} \mathrm{C}$, the surface is covered by nanoscopic filaments with diameter lower than $100 \mathrm{~nm}$ (fig 6c). At $710^{\circ} \mathrm{C}$, the filament size increases to about $200-250 \mathrm{~nm}$, while at $1200^{\circ} \mathrm{C}$ a rounded morphology is observed with a characteristic scale around $500 \mathrm{~nm}$. The cross-section images show the presence of voids in the near surface region, the typical size of which increases with the surface temperature. It is interesting to notice that the characteristic dimensions of the surface morphology correlates well with the size of the near-surface voids- confirming that both are linked. The size distribution of the voids is very large and ranges, for the highest temperature, from several $\mathrm{nm}$ to $500 \mathrm{~nm}$ (fig. 6d). The voids are inhomogeneously distributed within the sub-surface region. A sharp boundary exists between the plasmamodified area and the undamaged bulk material. The thickness of the plasma-modified zone increases with the surface temperature similarly to the size of the near-surface voids. Fig. 7 summarizes the evolution of the thickness of the plasma-modified layer as a function of the surface temperature and plasma exposure time. Since the samples were exposed under similar ion flux conditions, the latter is equivalent to the ion fluence. The nano-structured layer thickness evidently increases with surface temperature and exposure time. A factor 3 increase of the thickness is observed between $710^{\circ} \mathrm{C}$ and $1200^{\circ} \mathrm{C}$. In addition, when the exposure time is doubled (500 s and $1000 \mathrm{~s}$ ), the thickness increases by a factor 2.2 and 2.7 at $710^{\circ} \mathrm{C}$ and $1200^{\circ} \mathrm{C}$, respectively.

Fig. 8 illustrates the effect of the surface temperature on the helium-induced morphology changes of tungsten surfaces bombarded by $45 \mathrm{eV}$ helium ions. As for the case of molybdenum surfaces (fig. 6), there is a strong correlation between the surface temperature during plasma exposure and the resulting structure size. At $1000^{\circ} \mathrm{C}$, nanoscopic filaments with diameter lower than $20 \mathrm{~nm}$ are formed. When the temperature is increased to $1500^{\circ} \mathrm{C}$, the structure size is in the range 100-200 nm while micrometric voids are observed on the surface of the sample exposed at $2000^{\circ} \mathrm{C}$. The cross-section images are also consistent with those of the molybdenum samples with an increase in the size of the near-surface voids with 
surface temperature. It should be mentioned that voids are not visible in the picture shown here for the sample exposed at $1000^{\circ} \mathrm{C}$, because of their very small size. As in the case of molybdenum surfaces, a sharp boundary exists between the plasma-modified region and the unaffected bulk.

In contrast to the case of molybdenum surfaces, the kinetic development of heliuminduced nano-structures has been previously studied by Baldwin et $\mathrm{al}^{6}$ for temperatures of $850^{\circ} \mathrm{C}$ and $1050^{\circ} \mathrm{C}$. The thickness of the nanostructured layer was found to follow a Fick's law (i.e. $\mathrm{d} \mathrm{t}^{1 / 2}$ ) with an activation energy of $0.71 \mathrm{eV}$. A similar relationship was established by Kajita et $\mathrm{al}^{21}$ for slightly higher surface temperatures $\left(1130^{\circ} \mathrm{C}\right)$. The latter study however concluded to an effective diffusion coefficient which is actually intermediate between the values derived at $850{ }^{\circ} \mathrm{C}$ and $1050{ }^{\circ} \mathrm{C}$ in ${ }^{6}$. It should be mentioned that in those two references, the plasma exposure is made in a single step while the results in this study have been obtained with successive plasma exposures with a duration of $100 \mathrm{~s}$ - the surface morphologies being actually similar in the three different studies.

The temporal evolution of the nano-structured layers produced, on tungsten, in this study is plotted in figure 9 for three different surface temperatures- corresponding to the samples shown in fig. 8. In order to compare the present results with previously reported data, the measured structure thickness is reported as a function of $t^{1 / 2}$. The dashed lines represent the growth rate observed in ${ }^{6}$ for the cases of $850{ }^{\circ} \mathrm{C}$ and $1050{ }^{\circ} \mathrm{C}$ (experimentally measured), while an extrapolation to $1200{ }^{\circ} \mathrm{C}$ of these results assuming the measured activation energy of $0.71 \mathrm{eV}$ is also plotted. The measured nano-structure thickness at $1200{ }^{\circ} \mathrm{C}$ is in good agreement with the expected thickness while significantly lower growth rates are observed at higher temperatures. For instance, the growth rate obtained for a $500 \mathrm{~s}$ exposure $\left(\mathrm{t}^{1 / 2}=22.4 \mathrm{~s}^{1 / 2}\right)$ is decreased by a factor 2 when the surface temperature is increased from $1200^{\circ} \mathrm{C}$ to $1400^{\circ} \mathrm{C}$. On the other hand, the final thickness after $1000 \mathrm{~s}$ of exposure is similar for temperatures of $1400^{\circ} \mathrm{C}$ and $2000^{\circ} \mathrm{C}$. The exposure times in the present study are on the low side of those in ${ }^{6,21}$ where up to 100 times higher exposure times are used. The restricted exposure time range might increase the observed deviation from previous results. The studies by Baldwin et al have been done at temperatures below $1050^{\circ} \mathrm{C}$ while the two other studies deal with surface temperature above $1200^{\circ} \mathrm{C}$. Re-crystallisation of tungsten starts in the temperature range $1200-1400^{\circ} \mathrm{C}$ (depending on the initial grade and preparation). For temperatures above $1200^{\circ} \mathrm{C}$, the reported growth rates are lower than that at 
$1050^{\circ} \mathrm{C}$ and below. At the same time, however, the void size increases strongly with temperature which has been described $i^{22}$ albeit in the temperature range $730-1160^{\circ} \mathrm{C}$. Also, studies of desorption of implanted helium from tungsten surfaces have shown the presence of desorption peaks at 730,1230 and $1930^{\circ} \mathrm{C}^{17,23}$. There is therefore an interplay between helium bubble formation, coalescence, re-crystallization and desorption influencing the final surface structure.

Transmission Electron Microscopy was used to gain more insight into the inner structure of the tungsten filaments. Fig. 10 shows TEM pictures of tungsten filaments collected from the surface of a plasma-exposed tungsten sample exposed at $1000^{\circ} \mathrm{C}$ for a duration of $500 \mathrm{~s}$ (fluence $=3.2 \times 10^{26} \mathrm{~m}^{-2}$ ). The arborescent nature of the nanostructure is evident from fig. 10a. A large number of nanometric bubbles can be observed inside the filaments (fig. 10b) with a wide size distribution and sizes lower than $20 \mathrm{~nm}$. The voids present irregular shapes with facetted structures. No spherical voids can be observed. The interference patterns between the transmitted and diffracted electron beams reveal the presence of parallel atomic planes in the filament core, indicating a crystalline structure (fig. 11). The structure is similar to that of $\alpha$-tungsten, which is the stable form of tungsten.

\section{CONCLUSIONS}

Our results show that nanostructure formation on molybdenum and tungsten by low energy helium ions proceeds along the same scheme and depends on similar experimental parameters namely the ion energy, surface temperature and exposure time. The size of the resulting structure is mostly dependent on the surface temperature which is thus the most critical parameter for the control of the filament size and structure porosity. It has previously be demonstrated ${ }^{10}$ that structures similar to that shown in fig. $8 \mathrm{c}$ and $\mathrm{f}$, had a reflectance lower than $1 \%$ across the visible spectrum. The results presented here show that the formation of such highly porous structures can be realized on both molybdenum and tungsten surfaces by low-energy helium ion irradiation.

\section{REFERENCES}

${ }^{1}$ R. van de Krol, Y. Liang, and J. Schoonman, J. Mater. Chem., 18, 2311 (2008) 
${ }^{2}$ J. Su, L. Guo, N. Bao, and A.C. Grimes, Nano Letters, 11, 1928 (2011)

${ }^{3}$ E. Rephaeli and S. Fan, Appl. Phys. Lett., 92, 211107 (2008)

${ }^{4}$ K. Tokunaga, M.J. Baldwin, R.P. Doerner, N. Noda, Y. Kubota, N. Yoshida, T. Sogabe, T. Kato, B. Schedler, J. Nucl. Mater., 337-339, 887 (2005)

${ }^{5}$ S. Takamura, N. Ohno, D. Nishijima, and S. Kajita, Plasma Fusion Res. 1, 51, (2006)

${ }^{6}$ M.J. Baldwin, and R.P. Doerner, Nucl. Fus., 48035001 (2008)

${ }^{7}$ S. Kajita, W. Sakaguchi, N. Ohno, N. Yoshida, and T. Saeki, Nucl. Fusion, 49, 095005 (2009)

${ }^{8}$ S.I. Krasheninnikov, Phs. Scr. T145, 014040 (2011)

${ }^{9}$ M.J. Baldwin, and R.P. Doerner, J. Nucl. Mater., 404, 165 (2010)

${ }^{10}$ S. Kajita, T. Saeki, N. Yoshida, N. Ohno, and A. Iwamae, Applied Physics Express, 3, 085204, (2011)

${ }^{11}$ S. Kajita, T. Saeki, Y. Hirahata and N. Ohno, Japanese Journal of Applied Physics, 50, 01AH02 (2011)

${ }^{12}$ G.J. van Rooij and V.P. Vermiyenko and W.J. Goedheer and B. de Groot, A.W. Kleyn, P.H.M. Smeets. T.M. Versloot, D.G. Whyte, R. Engeln, D.C. Schram, and N.J. Lopes Cardozo, Appl. Phys. Lett., 90, 121501 (2007)

${ }^{13}$ H. J. van der Meiden and R. S. Al and C. J. Barth and A. J. H. Donne and R. Engeln and W. J. Goedheer and B. de Groot and A. W. Kleyn and W. R. Koppers and N. J. Lopes Cardozo and M. J. van de Pol and P. R. Prins and D. C. Schram and A. E. Shumack and P. H. M. Smeets and W. A. J. Vijvers and J. Westerhout and G. M. Wright and G. J. van Rooij, Rev. Sci. Instrum., 79, 013505 (2008)

${ }^{14}$ P.C. Stangeby, The Plasma Boundary of Magnetic Fusion Devices, Bristol and Philadelphia: Institute of Physics Publishing; 2002

${ }^{15}$ M.A. van den Berg, K. Bystrov, R. Pasquet, J.J. Zielinski, and G. De Temmerman, J. Nucl. Mater., submitted

${ }^{16}$ W. Sakaguchi, S. Kajita, N. Ohno, and M. Takagi, J. Nucl. Mater., 390-391, 1149 (2009)

${ }^{17}$ M.J. Baldwin and T.C. Lynch and R.P. Doerner and J.H. Yu, J. Nucl. Mater., 415, S104 (2011)

${ }^{18}$ K.O.E. Henriksson, K. Nordlund and J. Keinonen, Nuclear Instruments and Methods in Physics Research, B244, 377 (2006)

${ }^{19}$ K J Close and J Yarwood, British Journal of Applied Physics, 18, 1593 (1967) 
${ }^{20}$ M.J. Baldwin and R.P. Doerner and D. Nishijima and K. Tokunaga and Y. Ueda, J. Nucl. Mater., 390-391, 886 (2009)

${ }^{21}$ S. Kajita, N. Yoshida, R. Yoshihara, N. Ohno and M. Yamagiwa, J. Nucl. Mater., 418, $152(2011)$

${ }^{22}$ S. Sharafat, A. Takahashi, Q. Hu, and N.M. Ghoniem, J. Nuc. Mater., 386-388, 900 (2009)

${ }^{23}$ E.V. Kornelsen, and A.A. van Gorkum, J. Nucl. Mater., 92, 79 (1980) 
Figure 1: Typical electron temperature $\left(\mathrm{T}_{e}\right)$ and density $\left(\mathrm{n}_{e}\right)$ profiles of the helium plasmas used during the experiments. The FWHM of the plasma beam is typically $1 \mathrm{~cm}$.

Figure 2: Evolution of the surface temperature and emissivity of (a) tungsten and (b) molybdenum surfaces exposed to high flux helium plasmas $\left(1-2 \times 10^{24} m^{-2} s^{-1}\right)$ with $\mathrm{E}_{\text {ion }}=45 \mathrm{eV}$

Figure 3: Surface morphology of molybdenum surfaces induced by helium plasma exposure for a duration of $500 \mathrm{~s}$, with a surface temperature of $1000^{\circ} \mathrm{C}$ as a function of the impinging ion energy.

Figure 4: Surface morphology of tungsten surfaces induced by helium plasma exposure for a duration of $500 \mathrm{~s}$, with a surface temperature of $1000^{\circ} \mathrm{C}$ as a function of the impinging ion energy.

Figure 5: Scanning electron microscope images of tungsten surface after exposure to a helium fluence of $3 \times 10^{26} \mathrm{~m}^{-2}(500 \mathrm{~s})$ with a surface temperature of $1400^{\circ} \mathrm{C}$, with $E_{\text {ion }}=$ $25 \mathrm{eV}$ (a and b- with a tilt of $45^{\circ}$ ) and $E_{i o n}=45 \mathrm{eV}$ (c and d-cross-section images)

Figure 6: Evolution of the helium-induced surface morphology changes of molybdenum surfaces as a function of the surface temperature during plasma exposure $\left(\mathrm{E}_{i o n}=45 \mathrm{eV}\right.$, $\mathrm{t}=1000 \mathrm{~s}$ ). For each exposure temperature, top-views (tilted by $52^{\circ}$ ) and cross-sections are shown in the same magnification on the left and right sides, respectively. The dashed line in (d) indicates the boundary between the plasma-modified layer and the unaffected bulk.

Figure 7: Evolution of the thickness of the plasma-modified sub-surface region as a function of the surface temperature during exposure for molybdenum surfaces. The ion energy was $45 \mathrm{eV}$.

Figure 8: Evolution of the helium-induced surface morphology changes of tungsten surfaces as a function of the surface temperature during plasma exposure $\left(\mathrm{E}_{i o n}=45 \mathrm{eV}\right)$. For each temperature, top-views and cross-sections are shown on the left and right sides, respectively. The exposure duration was $1000 \mathrm{~s}$ for the samples prepared at $1500^{\circ} \mathrm{C}$ and $2000^{\circ} \mathrm{C}$, and $500 \mathrm{~s}$ for the sample prepared at $1000^{\circ} \mathrm{C}$.

Figure 9: Evolution of the thickness of the nanostructured region as a function of exposure time for tungsten surfaces, with $\mathrm{E}_{\text {ion }}=45 \mathrm{eV}$.

Figure 10: Transmission electron microscope images of tungsten filaments formed on a tungsten surface during helium plasma exposure. The surface was exposed at $1000^{\circ} \mathrm{C}$, for $500 \mathrm{~s}$ with $\mathrm{E}_{\text {ion }}=45 \mathrm{eV}$. (a) and (b) are images of the same area with different magnifications. 
Figure 11: Electron diffraction pattern of the tungsten filaments with hkl identification. 


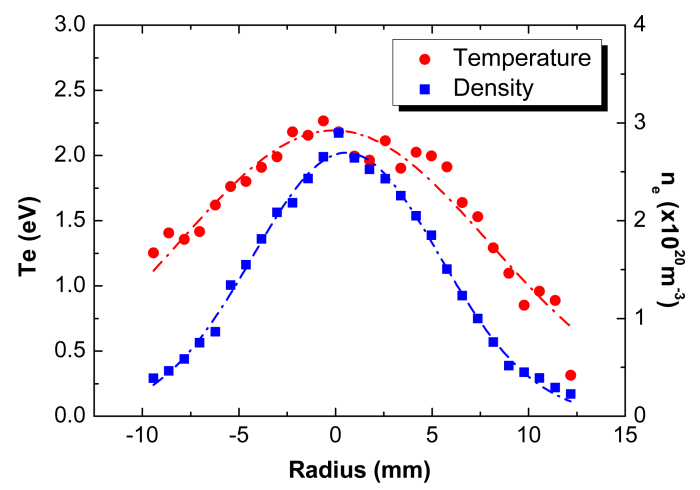

FIG. 1. (Color online) Typical electron temperature $\left(\mathrm{T}_{e}\right)$ and density $\left(\mathrm{n}_{e}\right)$ profiles of the helium plasmas used during the experiments. The FWHM of the plasma beam is typically $1 \mathrm{~cm}$. 

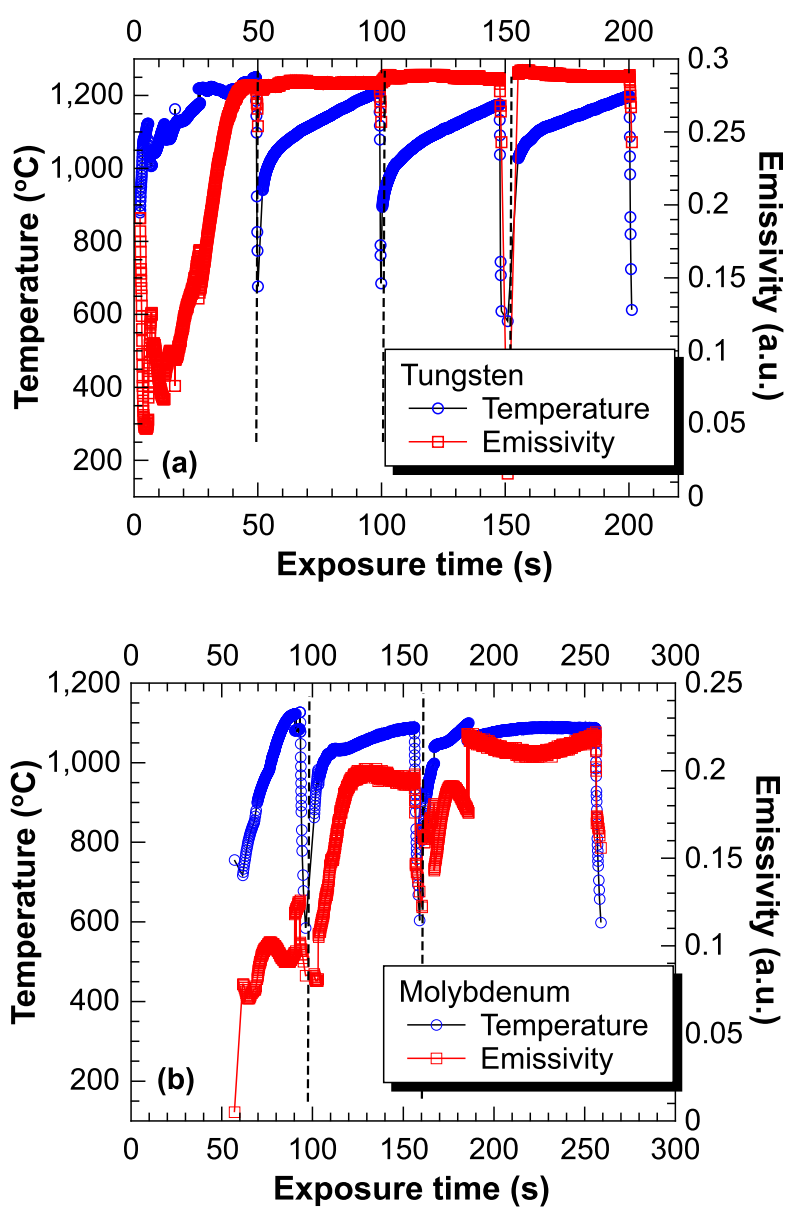

FIG. 2. (Color online) Evolution of the surface temperature and emissivity of (a) tungsten and (b) molybdenum surfaces exposed to high flux helium plasmas $\left(1-2 \times 10^{24} m^{-2} s^{-1}\right)$ with $\mathrm{E}_{i o n}=45 \mathrm{eV}$ 


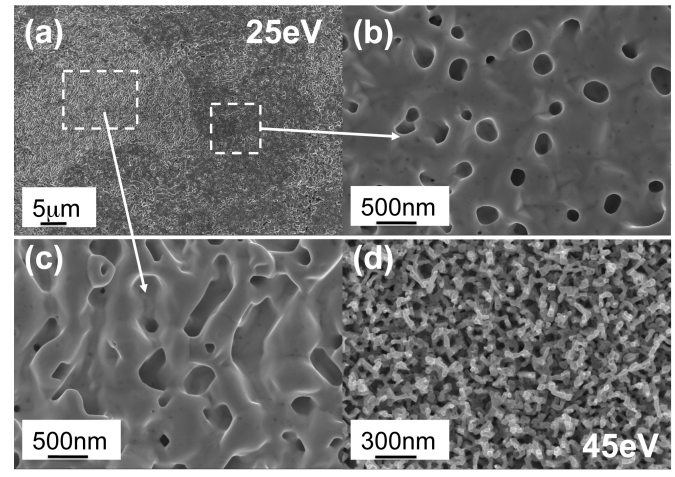

FIG. 3. Surface morphology of molybdenum surfaces induced by helium plasma exposure for a duration of $500 \mathrm{~s}$, with a surface temperature of $1000^{\circ} \mathrm{C}$ as a function of the impinging ion energy. 


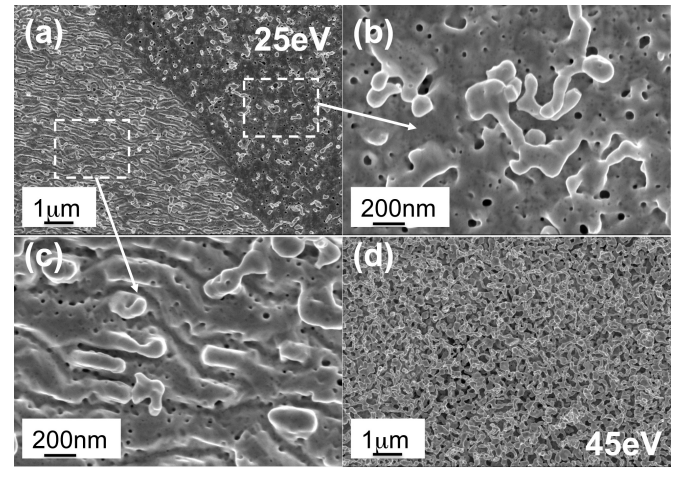

FIG. 4. Surface morphology of tungsten surfaces induced by helium plasma exposure for a duration of $500 \mathrm{~s}$, with a surface temperature of $1000^{\circ} \mathrm{C}$ as a function of the impinging ion energy. 


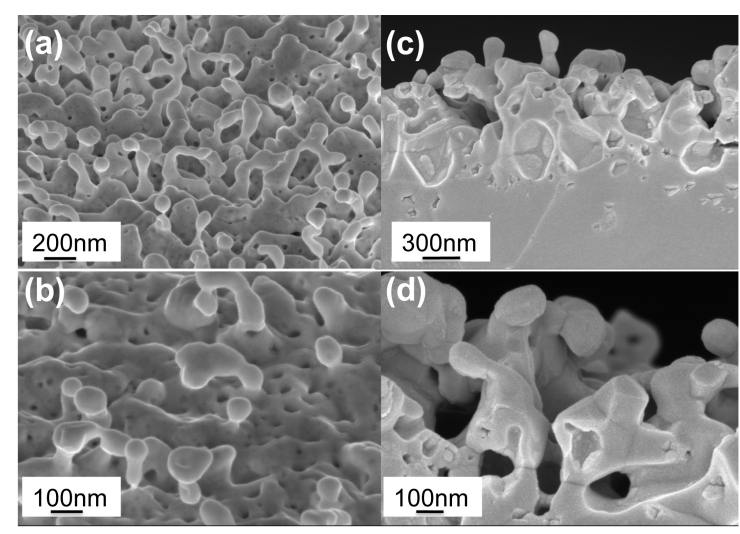

FIG. 5. Scanning electron microscope images of tungsten surface after exposure to a helium fluence of $3 \times 10^{26} \mathrm{~m}^{-2}(500 \mathrm{~s})$ with a surface temperature of $1400^{\circ} \mathrm{C}$, with $E_{i o n}=25 \mathrm{eV}$ (a and b- with a tilt of $45^{\circ}$ ) and $E_{i o n}=45 \mathrm{eV}$ (c and d- cross-section images) 


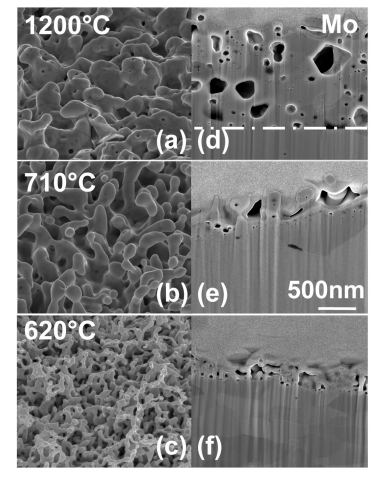

FIG. 6. Evolution of the helium-induced surface morphology changes of molybdenum surfaces as a function of the surface temperature during plasma exposure $\left(\mathrm{E}_{i o n}=45 \mathrm{eV}, \mathrm{t}=1000 \mathrm{~s}\right)$. For each exposure temperature, top-views (tilted by $52^{\circ}$ ) and cross-sections are shown in the same magnification on the left and right sides, respectively. 


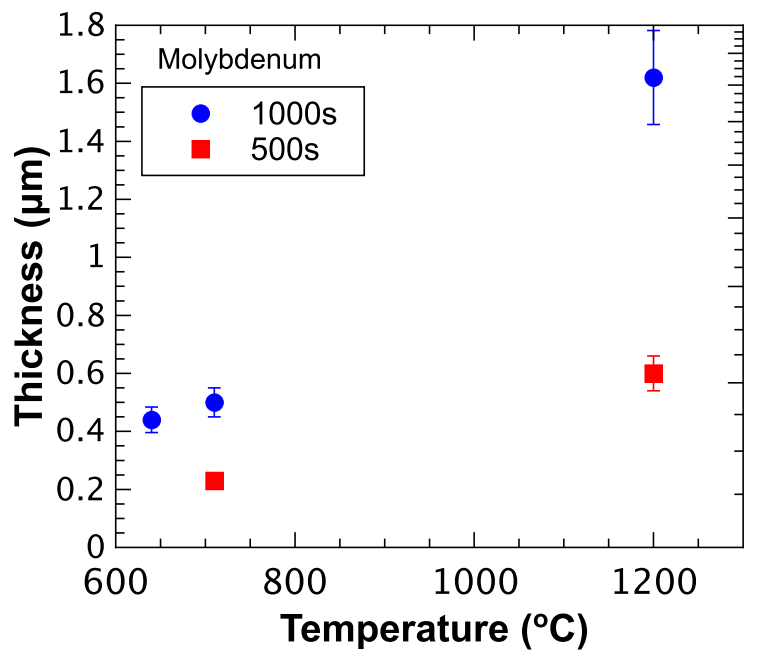

FIG. 7. (Color online) Evolution of the thickness of the plasma-modified sub-surface region as a function of the surface temperature during exposure for molybdenum surfaces. The ion energy was $45 \mathrm{eV}$, the ion fluence was $4.5 \times 10^{26} \mathrm{~m}^{-2}(500 \mathrm{~s})$ and $9 \times 10^{26} \mathrm{~m}^{-2}(1000 \mathrm{~s})$. 


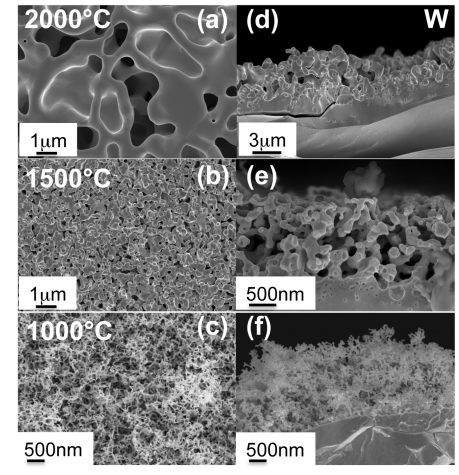

FIG. 8. Evolution of the helium-induced surface morphology changes of tungsten surfaces as a function of the surface temperature during plasma exposure $\left(\mathrm{E}_{i o n}=45 \mathrm{eV}\right)$. For each temperature, top-views and cross-sections are shown on the left and right sides, respectively. The exposure duration was $1000 \mathrm{~s}$ for the samples prepared at $1500^{\circ} \mathrm{C}$ and $2000^{\circ} \mathrm{C}$, and $500 \mathrm{~s}$ for the sample prepared at $1000^{\circ} \mathrm{C}$. 


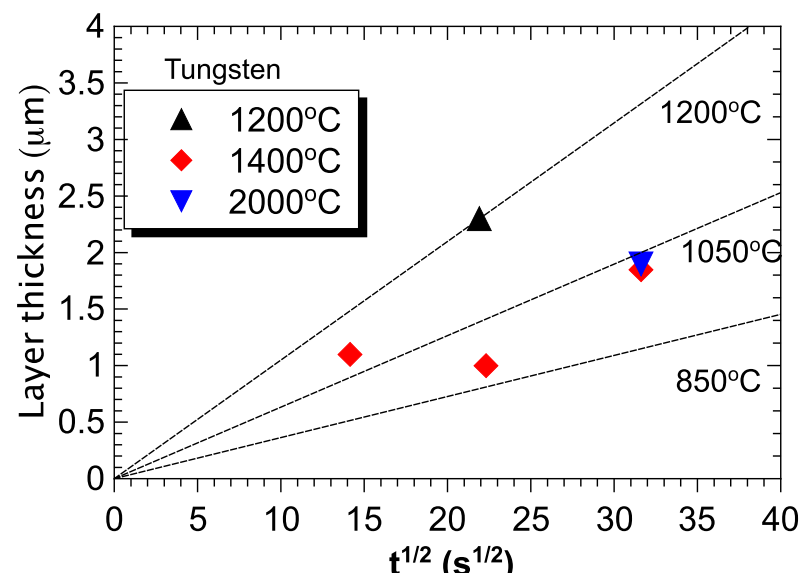

FIG. 9. (Color online) Evolution of the thickness of the nanostructured region as a function of exposure time for tungsten surfaces, with $\mathrm{E}_{\text {ion }}=45 \mathrm{eV}$.

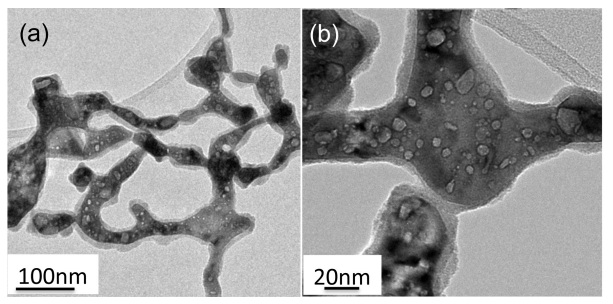

FIG. 10. Transmission electron microscope images of tungsten filaments formed on a tungsten surface during helium plasma exposure. The surface was exposed at $1000^{\circ} \mathrm{C}$, for $500 \mathrm{~s}$ with $\mathrm{E}_{\text {ion }}=45 \mathrm{eV}$. (a) and (b) are images of the same area with different magnifications. 


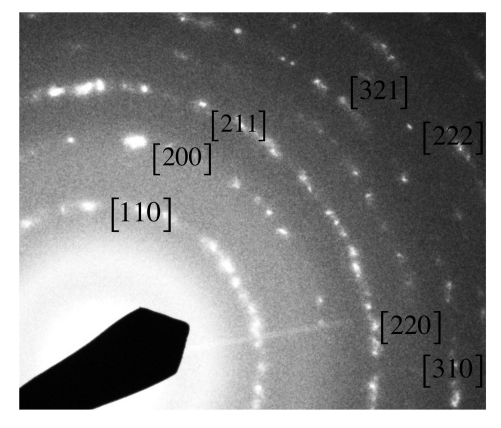

FIG. 11. Electron diffraction pattern of the tungsten filaments with hkl identification. 